\title{
Development of Gas Well Opening Device for Remote Control
}

\author{
Yong Chen ${ }^{1, a^{*}}$, Xiaohong Bai ${ }^{2, b}$ and Yaode $\mathrm{Li}^{1, \mathrm{c}}$ \\ ${ }^{1}$ Oil \& Gas Technology Research Institute, Changqing Oilfield Company, \\ Xi'an,Shaanxi710018,China \\ ${ }^{2}$ Low-permeability oil and gas exploration and development of National Engineering Laboratory, \\ Xi'an,Shaanxi710018,China \\ a187055127@qq.com, bxh_cq@126.com, ${ }^{\mathrm{c}}$ lyd_cq@126.com
}

Keywords: gas field; depressurizing; remote control; valve

Abstract. With an area of more than forty thousands square kilometers, rich geological reserves have been proved in Sulige gas field and thousands of gas wells have been constructed there. It is a high cost to open or shut in so many wells manually and labour intensity is great for workers. To meet the requirements of matching technologies and management methods for Sulige gas field development, devices of gas well opening and shutting in for remote control were developed before, which could implement the functions of emergency cutting off and opening well within a limit of low pressure. This paper introduced the development of a new type of gas well opening device for remote control, which could open gas wells at high pressure. The whole structure, working principle and design of key parts are introduced. Meanwhile, field tests were developed. The device this paper developed could realize the function of opening wells in wide range of pressure remotely, and could improve the level of automation of gas field.

\section{Introduction}

With an area of more than forty thousands square kilometers, rich geological reserves have been proved in Sulige gas field and thousands of gas wells have been constructed there. But most of the wells are were located in desert or mountain area with hardship roads and long distances, and nature environments are adverse there. With the enlarging devoloping scale of Sulige gas field, more and more production wells will be constructed, and the total is up to ten thousands in the near future. It is a high cost to open or shut in so many wells manually and labour intensity is great for workers. Hence, opening or shutting in gas wells remotely is the future direction for the management of Sulige gas field.

Gathering pattern of medium and low pressure is adopted in Sulige gas field and technologies of downhole choke are widely used which could depress the pressures of well heads. Thus, there are two pressure systems between bottom hole and ground, and pipelines of medium pressure are designed above ground [1]. In the event of invalidation of downhole choke, high pressure will occur at well head, which is much higher than the designed pressure of ground pipeline of $6.3 \mathrm{MPa}$. To avoid the hidden danger, devices of gas well opening and shutting in for remote control were developed before, which could realize the functions of emergency cutting off. Meanwhile, another function of opening well remotely within a limit of low pressure is applied [2]. However, in the stage of high pressure production, they could not be open remotely. Workers must go to the well sites and operate the needle valve manually to control the pressure of pipeline in safety range.

This paper introduced the development of a new type of gas well opening device for remote control, which could open gas wells at high pressure. The whole structure, working principle and design of key parts are introduced. The device this paper developed could realize the function of opening wells in wide range of pressure remotely. Manual operations of well opening at well sites could be replaced and the level of automation of gas field could be improved. 


\section{Structure Design of Gas Well Opening Device for Remote Control}

Design Requirements Aiming at the environment and facilities in Sulige gas field, 12V DC supplies with solar power generation are available. Meanwhile, the device should be designed with a characteristic of self-holding to avoid the hidden danger to the gas production brought by the long-term electrified electromagnetism coil. The actions of open the wells or cut off the pipelines should be triggered by weak current.

The Whole Structrue The gas well opening device for remote control is mainly comprised of valve core sleeve, decompression component, full scale opening component and mini electromagnetic valve etc. The concrete structure is shown as Fig.1.
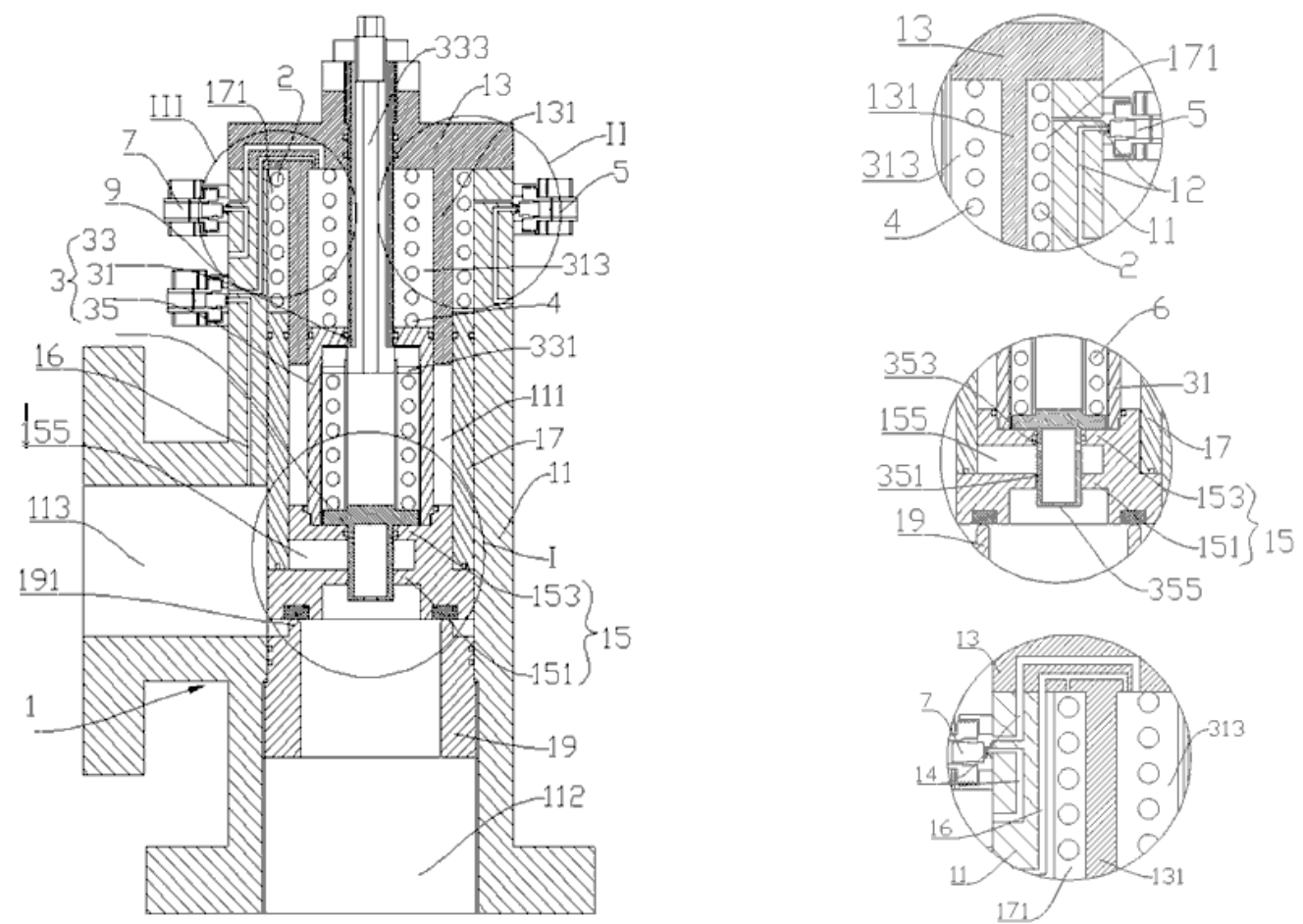

1. gas well opening device for remote control,2. 1st elastic element,3. decompression component,4. 2nd elastic element,5. 1st mini electromagnetic valve,6. 3rd elastic element,7. 2nd mini electromagnetic valve,9. 3rd mini electromagnetic valve,11. valve body,12. 1st pressure relief channel,13-valve cover,14. 2nd pressure relief channel,15. valve core,16. pressurizing channel,17. valve core sleeve,19. valve seat,31. body of decompression valve,33. pressure adjusting rod,35. core of decompression valve,111. cavity,112. outlet channel,113. inlet channel,131. fixing sleeve, 151 . supporting bench,153. mounting bench,155. gap,171. upper cavity of valve core sleeve,191-supporting convex loop,313. upper cavity of valve core,331. pressure adjusting nut,333. through-hole,351.cylinder,353.inlet hole,355. outlet hole

Fig. 1 The concrete structure of gas well opening device for remote control

The valve core sleeve is the barrier of the valve core and the switch of the decompression component, by which the gas flow channel is controlled. The decompression component could ensure that the pressure of the downstream pipeline is in safety range. The full scale opening component could lift up the whole mechanism and open the gas flow channel entirely to avoid throttle effect. The mini electromagnetic valves could open or cut off the inner gas channels according to the remote control signal with the aim of driving the components to fulfill functions corresponding to.

Working Principle As shown in figure 1, electrify 1st mini electromagnetic valve and the mandrel will retract. So, the terminal seal surface of the mandrel is opened and pressure relief channel of the device is unobstructed. At the moment, the upper cavity is connected with the outer atmosphere and the inner pressure there drop sharply. Under the effect of pressure difference, the valve core sleeve is lifted. Meanwhile, the gas flows in the decompression channel and flow out via outlet hole. Thus, the decompression component works.

Some time elapse and the pressure of the inlet channel will drop result from the pressure dropping underground. The 2 nd mini electromagnetic valve will be electrified by the digitization control system 
as soon as the pressure of inlet channel is close to that of outlet channel. So, the mandrel will retract. The terminal seal surface of the mandrel is opened and 2nd pressure relief channel of the device is unobstructed. At the moment, the upper cavity of the decompression component is connected with the outer atmosphere and the inner pressure there drop sharply. Under the effect of pressure difference between the bottom surface of the full scale opening component and upper end surface of that, all the components are lifted as a whole. Therefore, the inlet channel and outlet channel are clear, and the device is opened entirely. The process of opening well under the condition of high pressure with remote control device finished.

When there is a need to shut in the well, the self-holding of the the1st and 2 nd mini electromagnetic valve will be interrupt by the digitization control system, and the mandrel of them will return to the former places and the pressure relief channel will be cut off by the terminal seal surface of the mandrel. At the same time, the 3rd mini electromagnetic valve will be electrified. the mandrel will retract. The terminal seal surface of the mandrel is opened and pressurizing channel of the device will be connected. At the moment, the pressure of the upper cavity of the valve core and valve core sleeve increase sharply. Then all of the components drop away from the valve cover under the effect of pressure difference, force of gravity and elastic force of elastic elements. Thus, outlet channel is cut off. The process of shutting in the well with the remote control device finished.

\section{Design and calculation of Key Parts}

Design of Valve Core Sleeve Component The valve core sleeve component is the barrier of the valve core and the switch of the decompression component, by which the gas flow channel is controlled. Also, impact resistance is one of the key factors in the design. The valve core sleeve component is mainly comprised of body of valve core sleeve, special shaped seal ring, gland, Y-shape seal ring, mechanical spring, and disc spring. The design drawing of the valve core sleeve component is shown as Fig.2.

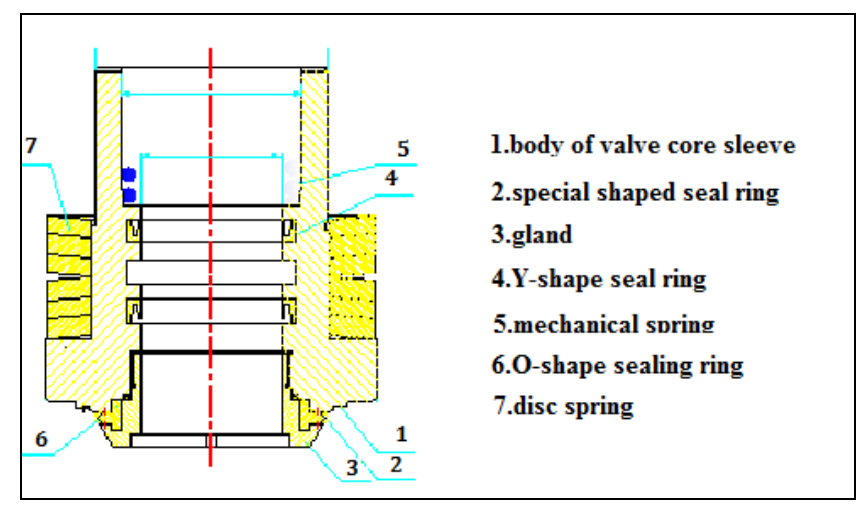

Fig. 2 The design drawing of the valve core sleeve component

The valve core sleeve is divided into two cavities by the elements-i.e. upper cavity and lower cavity. When gas flow in both of the cavity, the valve core sleeve will be pushed to the bottom end and device will keep off state. So, the device is of normal close.

Calculation and selection of the Cushioning Mechanism Considering the impact resistance of valve core sleeve, the cushioning mechanism is calculated. External diameter of valve core sleeve $\mathrm{D}=100 \mathrm{~mm}$, inner diameter $\mathrm{d}=60 \mathrm{~mm}$, stress area as following:

$$
\mathrm{S}=\frac{\pi}{4}\left(D^{2}-\mathrm{d}^{2}\right)=\frac{\pi}{4}\left(100^{2}-60^{2}\right)=5024 \mathrm{~mm}^{2}
$$

Static force of valve core sleeve when opening under different pressures are shown in Table 1. 
Table 1 Static force of valve core sleeve under different pressures

\begin{tabular}{c|c|c|c|c|c}
\hline External diameter D[mm] & 100 & 100 & 100 & 100 & 100 \\
\hline Inner diameter d[mm] & 60 & 60 & 60 & 60 & 60 \\
\hline Pressure P[Mpa] & 4 & 10 & 16 & 20 & 25 \\
\hline Opening force F[N] & 20096 & 50240 & 80384 & 100480 & 125600 \\
\hline Stressed Inner diameter[mm] & 78 & 78 & 78 & 78 & 78 \\
\hline Stress[Mpa] & 6.54 & 16.34 & 26.15 & 32.69 & 40.86 \\
\hline
\end{tabular}

From Table 1, it can be seen that maximum impact force of the valve cover is $125600 \mathrm{~N}$, and maximum stress is $40.858 \mathrm{MPa}$.

Velocity values of valve core sleeve at maximum stroke under different pressures are shown in Table 2.

Table 2 Velocity values of valve core sleeve

Distance $\mathrm{L}=18 \mathrm{~mm}$, Mass $\mathrm{m}=5.2 \mathrm{Kg}$

\begin{tabular}{c|c|c|c|c|c}
\hline Pressure P[Mpa] & 4 & 10 & 16 & 20 & 25 \\
\hline Opening force $\mathrm{F}[\mathrm{N}]$ & 20096 & 50240 & 80384 & 100480 & 125600 \\
\hline Accelerated velocity a $\left[\mathrm{m} / \mathrm{s}^{2}\right]$ & 3864.62 & 9661.54 & 15458.46 & 19323.08 & 24153.85 \\
\hline Velocity v[m/s] & 11.80 & 18.65 & 23.59 & 26.37 & 29.49 \\
\hline
\end{tabular}

Impact stress values of valve core sleeve at maximum stroke under different pressures are shown in Table 3.

Table 3 Impact stress values of valve core sleeve

\begin{tabular}{c|c|c|c|c|c}
\hline \multicolumn{6}{c}{ Impact time $\mathrm{t}=0.0001 \mathrm{~s}$, Mass $\mathrm{m}=5.2 \mathrm{Kg}$, Stress area $\mathrm{s}=3074 \mathrm{~mm}^{2}$} \\
\hline Pressure P[Mpa] & 4 & 10 & 16 & 20 & 25 \\
\hline Velocity v[m/s] & 11.80 & 18.65 & 23.59 & 26.37 & 29.49 \\
\hline Impact stress[Mpa] & 199.53 & 315.48 & 399.06 & 446.16 & 498.82 \\
\hline
\end{tabular}

Mechanical properties of the material are as follows: Tensile Strength $\sigma_{b}=635 \mathrm{MPa}$, yield strength $\sigma_{0.2}=440 \mathrm{MPa}$. From the calculation results, it can be seen that Impact stress is higher than the static force by ten times and yield strength are overrange. Cushioning mechanism must be optimal selection and disc springs are selected for the characteristics of small deformation and high strength. The special method of lapping tablets is adopt and improve the bearing capacity by three times.

\section{Simulation Analysis and Field Test of the Gas Well Opening Device}

Simulation Analysis With the help of ANSYS software, pressure distribution of the gas well opening device is analyzed and is shown as Fig.3. According to the analysis result, it can be seen that pressure before the device is $20 \mathrm{MPa}$.After the process of throttling via the device, the outflow pressure is under $4 \mathrm{MPa}$ with slight fluctuation inside. Distribution of velocity field is analyzed is shown as Fig.4.

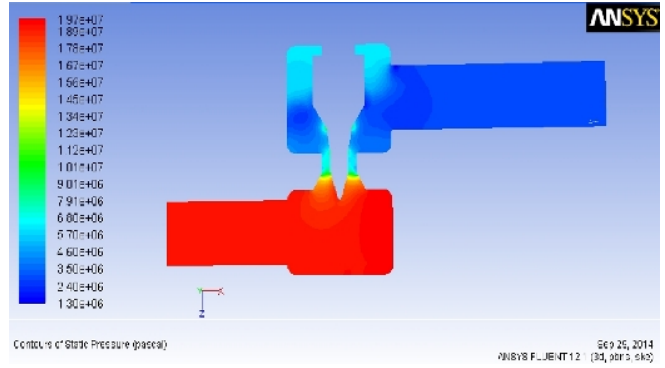

Fig.3 Analysis of pressure field distribution

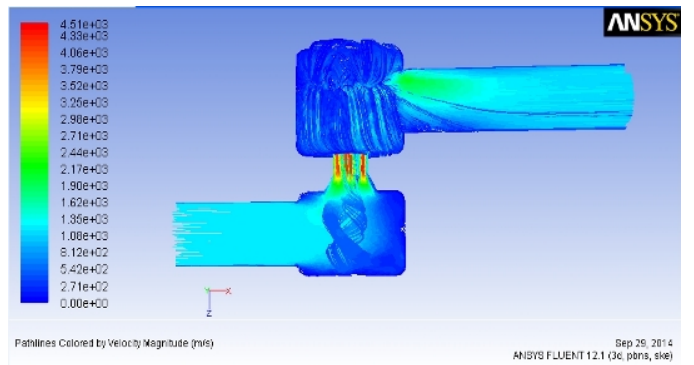

Fig.4 Analysis of pressure field distribution 
According to the analysis result of velocity field, it can be seen that the maximum of velocity occurred at the location of minimum area of passage. So, erosion resistance should be fully considered about the valve elements.

Field Test of the Gas Well Opening Device The field test were developed in Sulige gas field in November with ambient temperature of $5^{\circ} \mathrm{C}$. Tubing pressure of the test gas well is $20.68 \mathrm{MPa}$ before opening the device. When open the device by remote control, the pressure inflow dropped stably and the pressure outflow is lower than $4 \mathrm{MPa}$. The process of opening the well by the gas well opening device of remote control continued for about twenty minutes and opened the well successfully. The picture of the test is shown as Fig.5. The curve of opening the well is shown as Fig.6.

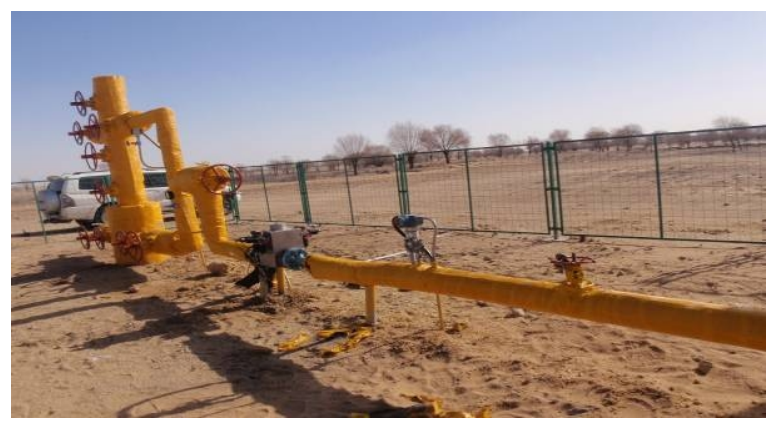

Fig.5 The picture of the test in Sulige gas field

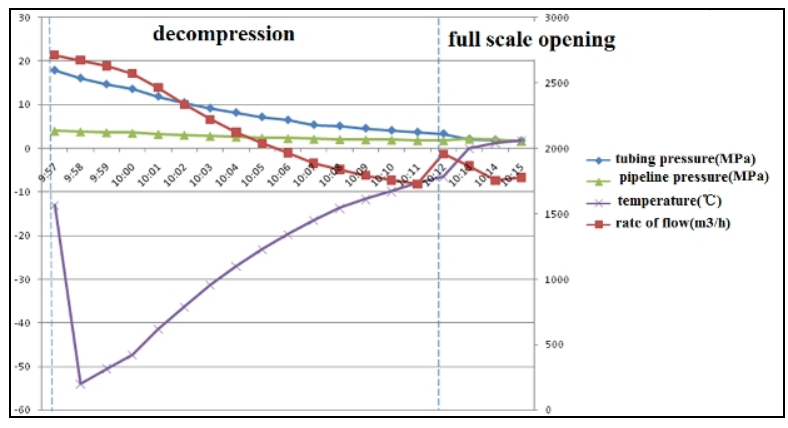

Fig. 6 The curve of opening the test well

\section{Conclusions}

(1) The gas well opening device for remote control this paper developed could realize the function of opening wells in wide range of pressure remotely. The process of opening gas well manually could be substitute for and the level of automation of gas field could be improved.

(2) The device devoloped possess the merits of energy conservation and safety, and could meet the future direction for management of Sulige gas field.

\section{Acknowledgements}

This work is supported by the National High Technology Research and Development Program (Project code: 2011AA040401). The authors appreciate the help of Senior Engineer Zhigang Yu for his support and thank all the workmates for their help with the work.

\section{References}

[1] X.Q. Ran, A.Q. Li, Sulige Gas Field Development Approach, Petroleum Industry Press, Bei Jing, 2008.

[2] Y. Chen, D. J. Chen, X. L. Cheng, Electromagnetic Valve of Remote Control for Gas Well, Oil Forum, 5(2012), 59-61. 